\title{
L'imitazione servile come atto di concorrenza sleale $(*)$
}

\author{
Mario Rotondi \\ Professore Ordinario di Diritto Commerciale- \\ nella Università di Pavía e nell'Università \\ Cattolica del S. Cuore di Milano.
}

1. II problema della "imitazione servile" in senso tecnico. - 2. La giurisprudenza e la dottrina tedesca e italiana. - 3. La imitazione servile e la Convenzione internazionale: l'art. 10 bis. -4 Illiceità della imitazione servile. - 5. La repressione dell'imitazione servile non significa equiparazione alla invenzione brevettata. - 6. Conclusione.

1 Tra gli svariati problemi che in materia di concorrenza sleale si pongono nei paesi industrialmente più progrediti, ve n'è uno che per la sua importanza pratica, e per

$\left(^{\star}\right)$ Il presente articolo ha particolare riferimento al conceito di concorrenza sleale introdotto nella Convenzione internazionale di Parigi alla Conferenza dell'Aja del 1925 (art. 10 bis) (rimasto sostanzialmente inalterato nella revisione della Conferenza di Londra del 1934), e al Codice civile italiano che riproduce con poche varianti la stessa norma (art. 2598). - Per il diritto brasiliano credo che l'ipotesi considerata in questo articolo potrebbe rientrare nella sanzione comminata dall' art. $178 \mathrm{n}$. III del Decreto Lei n. ${ }^{\circ}$ 7.903 del 27 agosto 1945. Comunque riterrei applicabile la generica e compreensiva formula dell art. 178 - Parágrafo unico, ai fini del risarcimento dei danni. 
la sua delicatezza teorica, pare dover reclamare in modo particolare l'attenzione dello studioso.

Il problema che si pone è questo: se la fabbricazione di un prodotto è coperta da un brevetto, la produzione che sia fatta da altri che non sia il titolare del brevetto o un suo autorizzato è certamente illecita, ed espone chi la compia alle gravi sanzioni civili e penali che le varie legislazioni comminano per la contraffazione. Ma se il prodotto non è coperto da privativa, sarà sempre lecita la sua produzione, anzi la sua riproduzione scrupulosamente fedele, senza il consenso e senza un compenso a chi ha speso denari, tempo, fatiche spesso ingentissimi per arrivare alla messa a punto di quel prodotto industriale?

La giurisprudenza ha ormai largamente ammesso che, ove l'imitazione dei caratteri esteriori del prodotto sia fatta per ingenerare confusione - o quando, anche indipendentemente da questo specifico scopo la confusione dei prodotti si renda possibile - l'attività del concorrente possa essere interdetta, e obbligo possa essergli fatto di differenziare la sua produzione per evitare equivoco o sviamento della clientela.

Ma quando la confusione sia esclusa, dovrà per ciò solo - in mancanza della protezione assoluta che deriva da un brevetto - considerarsi lecito il riprodurre magari con un semplice artificio meccanico il prodotto che fu faticosamente e costosamente realizzato da un altro?

Dato il carattere della produzione industriale moderna, che è diretta alla creazione di tipi standard, dati i requisiti rigorosi della invenzione brevettabile, che escludiono molte realizzazioni tecniche della protezione del brevetto, si comprende come il problema che si pone sia di eccezionale importanza pratica e si sia ormai ripetutamente posto alla giurisprudenza di diversi paesi.

Giova riconoscere che la soluzione lascia perplessi.

L'incertezza della soluzione è tale, che un autorevolissimo scrittore come il Piola-Caselli dopo di avere, come 
privato dottore, qualificata la imitazione servile dei modelli altrui "uno sfruttamento commerciale evidentemente ingiusto" (1) intervenendo come Procuratore Generale a prendere le conclusione avanti la Suprema Corte di Cassazione in una causa che rimase celebre, ha creduto di andare in contrario avviso (2).

Io sono personalmente convinto che l'imitazione "servile" del prodotto altrui, anche non protetto da privativa, debba essere condannata, sembrandomi ripugnare agli usi onesti dell'industria (art. 10 bis della Convenzione internazionale dello sfruttamente dei risultati del lavoro altrui (3). Ma credo che difficilmente - e fin qui è bene - la giurisprudenza possa arrivare alla condanna di una imitazione di un prodotto non brevettato - normalmente lecita certo - senza una esatta precisazione giuridica della sua qualifica di "servile".

Il precisare tale concetto per perseguire in ogni sua forma l'immoralità nei commenci è lo scoppo delle righe che seguono. L'applicarlo sanà compito della giurisprudenza, che nella dialettica dei contrastanti interessi fissa via via, con funzione altamente progressista, il principio del vero, e, quindi, del giusto.

2 La dottrina e la giurisprudenza germanica, che più hanno avuto occasione di occuparsi del problema - forse per il rigore dell'esame preventivo che restringe in Ger'mania i limiti della brevettabilità' hanno ammesso in vari casi che um prodotto, anche non coperto da privativa, non si possa lecitamente riprodurre se non entro i limiti

(1) v. Le cosi dette imitazioni servili nel sistema di protezione della proprietà intellettuale, in Annuario di diritto comparato, VII, Parte III, 1923), p. 269.

(2) v. Giur. Ital,. 1938. 1. 1. 433 in nota a Cass. 17 febbraio 1938.

(3) Iv. i miei precedente scritti in Giur. Ital. 1937. 127; Riv. di Dir. 1938. II. 99;

(4) Diritto industriale. ediz., Milano, 1942, p. 43 e segg. 
necessari al funzionamento tecnico; cioè entro i limiti della invenzione (cioè della soluzione del problema tecnico) in quanto questa non é tutelata dal diritto di esclusiva.

Tutto il resto, abbia semplice importanza formale od estetica, abbia utilità tecnica accessoria, se non è tutelato dalla privativa, e se della tutela mediante brevetto non ha nepure il presupposto - (se manca, vorremmo aggiungere con maggior rigore teorico, e seguendo la migliore dottrina, la res e la caratteristica difesa del diritto reale assoluto sull'invenzione - può avere però la tutela indïretta di natura non reale ma obbligatoria, fondata sulla repressione della concorrenza sleale.

La dottrina tedesca ha illustrata la casistica, ponendo in luce come la giurisprudenza del Reichsgericht derivi il principio della illiceità dal carattere servile della imitazione (sklavischer Nachbau), e richiamandosi allo sfruttamento illecito delle fatiche e delle spese altrui (Ausbeutung frem. der Muehe und Kostenaufwendung) (4). E sulla imitazione servile è sorta tutta una letteratura (5).

Secondo la precisa definizione dell'Isay deve intendersi per imitazione servile (sklavischer Nachbau) "quella in cui vengono copiate fedelmente nella forma e nelle dimensioni anche quelle parti della macchina le cui forme e dimensioni sono indifferenti pel funzionamento tecnico della macchina e per di più sono riprodotte con esattezza, nella forma e nelle dimensioni della macchina prodotta dal primo

(4) v.Reichsgericht 19 de marzo 1932; 12 febbraio 1929; 18 ottobre 1933 in ReIMER, Wettbewerbs und Warenzeicihenrecht, Berlino, 1935. II. p. $528-538$.

(5) cfr. Callmann, Der Unlautere Wettbewerb, Mannheim 1929, p. 120; e le monografie del Seligsohn, del CaHn, dello Spielmann, dell' Osterrieth, del Benjamin, del Frietze, dell Allfeld e di altri, e specialmente dell IsAy, Der Nachbau patentfreier Maschinen, in Gewerblicher Rechtsschutz und Urheberrecht, 1926, p. 83 e 253. 
fabbricante, anche quando forma e dimensioni possono, entro larghi estremi, essere liberamente variate" (6).

$\mathrm{E}$ dopo aver ricordata la decisione del Kammergericht del 3 dicembre 1924, che distingue appunto la riproduzione servile in sè, dalla riproduzione ai fini di creare confusione sull'origine del prodotto pur ritenendole entrambe illecite - l'Isay passa ad illustrare come la riproduzione servile sia riconosciuta illecita anche nel diritto inglese e nordamericano - (con famoso precedente delle macchine Singer) - per concludere che, se qualche diversità di punti di vista si può ravvisare nella giurisprudenza di questi paesi in confronto di quella germanica, anche per essi è "principio antico del diritto comune quello per cui ciascuno dev'essere difeso nei frutti del proprio lavoro e del proprio ingegno..." In una fattispecie decisa dall'Oberlandesgerich di Dresda (7) alcuni apparecchi separatori di latte denominati Alfa-Separatoren, erano stati imitati servilmente dal convenuto fino all'ultimo millimetro e messi in commercio sotto il nome di Hema Separatoren.

In altra fattispecie sottoposta all'esame del Kammergericht (8) erano state servilmente imitate le macchine originali Multigraph-Senior e Multigraph Junior ed erano state vendute col nome Dapag-Major e Dapag-Minor.

Particolarmente nella prima specie il giudice d'appello avrebbe forse potuto ammettere il pericolo di confusione, ma non lo ha fatto e ha piuttosto sostenuto che la imitazione sarebbe stata lecita soltanto nei limiti nei quali lo richiedesse la realizzazione dell'idea dĩ invenzione (caduta già nel dominio pubblico) non nei confronti di tutto quel complesso di dettagli costruttivi che rende servile l'imitazione. Nello stesso senso ha deciso il Kammargericht nella decisione surricordata dicendo che, anche senza il pericolo

(6) op. cit,. p. 83

(7) v. Musterschutz und Wettbewerb, XXIV. 5.

(8) v. Gewerblicher Rechtsschutz und Urheberrecht, 1925. 42. 
di confusione, la imitazione servile di una macchina non tutelata o non più tutelata dalla legge speciale, non sarebbe ssenz'altro lecita; tale imitazione sarebbe piuttosto una offesa grave ai buoni costumi se un concorrente sfruttasse in questa maniera le fatiche e le esperienze altrui, in quanto il loro impiego non è richiesto necessariamente dallo scopo della macchina in questione (9).

Nè la dottrina italiana è rimasta estranea al problema: basta ricordare il contributo di Alberto Parrella (10) che, attraverso esauriente esame della giurisprudenza italiana, tedesca e anglo-americana sulla imitazione servile, conclude per il carattere illecito di essa, indipendentemente dallo stesso concorso dell'elemento subbiettivo della colpa, e come attentato all'avviamento obbiettivo dell'azienda.

"Come nella complessità dei fenomeni morbosi, l'indagine clinica deve adeguarsi per sottigliezza di analisi alla più insidiose risorse del male, onde aprestare $i$ rimedi meglio adatti - ammonisce questo autore (11) — così nella varietà dei fenomeni sociali l'indagine giuridica deve pareggiare per penetrazione di ricerche, tutte le più ingegnose escogitazioni dell'egoismo, onde porre in essere le reazioni più idonee a contrastare le oscure aggressioni alla vita civile".

Il contributo prezioso della giurisprudenza in questa delicata materia, attinta ad una sagace e profonda disamina del caso, ha condotto ad una affermazione di grande portata che ha sconvolto ed abbattuto tutte le vecchie posizioni mentali, ed ha designato nella copia servile de prodotto dissociata d'a ogni elemento concomitante, il mezzo più pericoloso per attraversare il libero gioco delle com-

(9) cfr. anche la sentenza del Kammergericht in Gewerblicher Rechtsschutz und Urheberrecht, 1927.823.

(10) II divieto della copia servile nel sistema di difesa privata della proprietà industriale, in Studi in onore di Mariano D'Amelio, Roma, 1933, Vol. III, p. 17 segg.

(11) op. cit,. 17 
petizioni industriali. Ed il suo maggior merito è stato quello di aver chiarito alla evidenza come e perchè la repressione della copia servile, possa anche sopravvivere al venir meno della difesa del marchio e delle privative in genere (12).

Nè diversamente il Piola-Caselli occupandosi proprio dela imitazione servile, indipendentemente dal pericolo di confusione sull'origine del prodotto, scriveva: "che la teoria del divieto della imitazione servile non vada confusa con la teoria generale del divieto di quella forma di concorrenza che si esplica con mezzi atti a rendere equivoca: la provenienza del prodotto, risulta, anche da altre applicazioni che in questa teoria sono invocate, così, per es.,. nella nota questione della protezione della "moda di $\mathrm{Pa}$ rigi", Dopo i tentativi fatti specialmente in occasione della revisione di Roma della Convenzione di Berna, di pro-teggere la moda nella categoria delle opere dell'ingegno. che contempla le "opere d'arte applicate all'industria", si è invocata in recenti congressi l'arma del divieto della. concorrenza sleale, considerando come sleale, la imitazione: servile della moda di Parigi, fatta su modelli copiati... In questo caso, non si tratta evidentemente di equivoco di. provenienza industriale. Infatti la provenienza artistica è chiara; ma è chiaro da essa è diversa la provenienza industriale: il cliente sa bene che il modello è di Parigi, ma che è fabricato dal suo sarto. Le difficoltà tecniche speciali che si frappongono per realizzare in questi casi una privativa. regolare, con relative formalità di depositi di disegni 0 . modelli, sembrano appoggiare e giustificare la invocazione della teoria del divieto delle imitazioni servili, perimpedire uno sfruttamento commerciale che è evidentemen-te ingiusto (13).

(12) v. op. cit. p. 18

(13) Cfr. Annuario di diritlo comparato, vol. VII, Parte 3. ${ }^{\text {. }}$ (1933) p. 269. 
Anche nella giurisprudenza italiana il principio gia non ha mancato di trovare riconoscimento.

Cosi il Tribunale di Milano, con sentenza 6 ottobre 1928 . (14) affermava costituire concorrenza illecita: "il copiare il dispositivo e gli elementi di dettaglio dell'impianto concorrente - (notisi che si trattava di un impianto complesso di cui i singoli obbietti coperti da eventuale privativa non costituivano, che una piccola parte!) - quando anche non brevettato, e l'appropriarsi le particolari istruzioni di funzionamento dell'impianto stesso."

$\mathrm{E}$ non diversamente, ravvisando concorrenza sleale nella imitazione servile, insegnava la Suprema Corte con sentenza 26 gennaio 1932, (15), che chiariva anche come la imitazione servile riesca determinabile nella particolare somiglianza di un prodotto con altro determinato, a differenza che con tutti gli altri della stessa specie, la quale sentenza del Supremo Collegio dava modo alla Corte di Milano, adita in sede di rinvio, di precisare gli estremi della imitazione sleale con particolare riguando all'abuso di disegni e conoscenze derivanti da precorsi rapporti contrattuali (15).

Il nuovo ricorso della soccombente non serviva che a far ribadire a Sezioni Unite l'ormai pacifico ammestramento: (17) "Si sostiene dal ricorrente, rappresentando. l'opinione di una certa tendenza dottrinale, che sia incompatibile la repressione della imitazione sia pure servile di un prodotto quando questo sia diventato di dominio pubblico, o perche quel prodotto non sia brevettabile o non sia stato brevettato, o perchè sia scaduto il termine di durata del brevetto. $\mathrm{E}$ che non possa tale repressione

(14) in Riv. dir. comm,. 1932. II. 598.

(15) in Foro Ital,. 1932. 1. 868.

(16) w. App. Milano 20 maggio 1932, in Foro Ital,. 1932 I. 1274.-

(17) v. Cass. S. U. 8 giugno 1933 Pres. D'Amelio, Est. Parrella in Foro Ital,. 1933, 1.857. 
costituire una restrizione della libertà industriale cosi i!' mitata nel modo e nel tempo, da sovrapporsi al regolamento legale della privativa in guisa da tenderlo praticamente assorbito da essa e vuoto di pratico contenuto...

"La Corte non si indugiò oltre a designare le fonti di questa sua concezione giuridica, ma è agevole riconoscere che essa fece anche un riferimento alla teoria dell'atto illecito quale era stato già adombrato nella decisione della Cassazione.

"Il ragionamento si riannoda al principio generale del divicto della concorrenza sleale, ed una fonte ancora più specifica può riscontrarsi nell'art. 10-bis della Convenzione dell'Aja, ratificata col R. D. 10 gennaio 1926 n. 810 convertito in legge il 29 dicembre $1926 \mathrm{col} \mathrm{n} .271$ ed entrata in vigore al $1^{\circ}$. giugno 1928 , norma che considera atto di concorrenza sleale qualsiasi atto contrario agli usi onesti del commercio e qualsiasi atto capace di ingenerare la confusione dei prodotti. ."

Anche sucessivamente, con sentenza 1 dicembre 1933 (18) il Supremo Collegio riaffermava che costituisce illecita concorrenza il riprodurre servilmente la forma esteriore di un prodotto altrui".

E il Di Franco, commentando la stessa decisione (19) concludeva: "allorchè manchi la privativa, la imitazione sarà lecita entro i limiti di detti elementi intrinseci - (cioè quelli indispensabili per la funzione del prodiotto) - mentre sarà sempre reprimibile come atto di concorrenza sleale ove si estenda anche alla copia servile" (20)".

(18) v. Giur. Ital. 1934. 1. 1. 16.

(19) v.Foro Ital,. 1934. 1. 1526.

(20) Il Tribunale di Milano con sentenza 22 novembre 1934 (Mon. Trib. 1935.36) pur con riferimento all'elemento della confondibilità, dice "è da ritenere inoltre che il proposito malizioso e quasi fraudolento è insito nella stessa servilità dell' imitazione, allorché *questa si concreta nella imitazione di ogni dettaglio esteriore, anche insignificante o puramente ornamentale". 
3 Nè si dica che la imitazione servile è illecita soltanto in quanto sia strumento malizioso pier determinare una confusione di prodotti. Già più volte è stata sventata la assurda teoria che la confusione sia l'unico mezzo possible della concorrenza sleale (21) e non si può esitare conseguenzialmente a ritenere che la imitazione servile può essere mezzo di sleale concorrenza anche indipendentemente dallo scopo di creare confusione fra prodotti, sopratutto in quanto diretta nei confronti di un acquirente singolo, per bisogni peculiari del quale il prodotto è realizzato. Essa appare il prototipo di quegli atti contrari agli "usi onesti dell'industria" troppo spesso dimenticati, ma espressamente richiamati dall'art. 10-bis della Convenzione internazionale accanto agli "usi onesti del comercio" (22).

Senonchè da molti si concepisce l'imitazione servile come atto di concorrencia sleale solo in quanto atta a determinare confusione sull'origine dei prodotti, e si riferiscono gli infiniti casi di imitazione delle caratteristiche esteriori della confezione di un prodotto - bottiglie, involucri, carta di avvolgimento, astucci, etc. - di cui si vale spesso il concorrente sleale. Ma il prolema non finisce là: quasi che si potessero portare tutte le questioni di concorrenza sleale sul tavolo del pizzicagnolo; quasi

(21). V. sentenza 22 gennaio 1936, in Foro Ital. 1936. 1. 683.

(22) Secondo il testo riveduto a Londra l'art. 10 bis dice: "1. Les pays ide l'Union sont tenus d'assurer aux ressortissants de l'Union une protection effective contre la concurrence déloyale $\longrightarrow 2$. Constitue un acte de concurrence deloyale tout acte de concurrence contraire aux usages honnêtes en matière industrielle ou commerciale. 3. Notamment devront être interdits: $1 .^{\circ}$ tous faits quelconques de nature à créer une confusion par n'importe quel moyen avec l' établissement, les produits ou l'activité industrielle ou commerciale d'un concurrent; $2 .^{\circ}$ les allégations fausses, dans l'exercice du commerce, de nature à discréditer l'établissement, les produits ou l'activité industrielle ou commerciale d'un concurrent". 
che non esistesse imitazione se non per gli involucri di un surrogato di caffè!

In ciò si palesa una visione singolarmente angusta ed errata della concorrenza sleale per imitazione servile.

L'imitazione servile è atto di concorrenza sleale, e come tale repressa indipendentemente da ciò, e pel solo fatto che costituisce illegittima appropriazione del risultato del Iavoro, altrui, e quindi lesione di quello che con denominazione ormai corrente, si è detto avviamento obbiettivo dell'azienda, il cui titolare (23) attraverso la realizzazione di un prodotto tipico complesso, vede naturalmente la possibilita di uno smercio futuro che non deve essere compromesso dalla sleale manovra di terzi che pretendono di mietere dove non hanno seminato!

Quando sulla base dell'art. 10 bis della Convenzione internazionale si pretende di limitare la concorrenza sleale alle due forme della confusione e delle notizie false atte a screditare, si legge male l'articolo. Se queste due categorie di illecito sono espressamente previste e represse, esse non sono, come bene si palesa dalla lettera ("Notammente devront être interdits...") che una esemplificazione del più generale principio che condanna: "ogni atto contrario agli usi onesti del commercio e dell'industria".

Se la concorrenza sleale è la disonesta aggressione dell'avviamento del concorrente, giova ricordare che avviamento non è sinonimo di clientela. Diremmo che

(23) Sulla mia concezione dell'avviamento e sulla sua distinzione in avviamento obbiettivo (qualità dell'azienda dovuta ai singoli elementi materiali e immateriali e alla loro organizzazione) e avviamento soggettivo (dovuto alle qualità del titolarre dell'azienda) vedi i miei precedenti escritti: Trattato di diritto industriale, Padova, 1929, Vol. I, p. 153 segg.; Il diritto industriale, Milano, 1942, p. 57 segg.; Riv. dir. comm., 1928. I. p. 277 segg.; p. 463 segg.; 1929. I. p. 125; La nature juridique de lachalandage, in Annales de droit commercial, 1930, p. 1, 299 ultimo: El "aviamento" en la teoria general del fondo de comercio, in La Ley del 18 settembre 1948. 
questa non è che un riflesso esterno del primo, il quale $\dot{e}$ costituito da quella particolare speranza di lucri futuri che deriva ad un'azienda dai suoi stessi elementi obbiettivi, e dalla bontà tecnica e commerciale della sua organizzazione. Se l'imitazione servile può ledere la clientela, in quanto fonte di confusione sull'origine del prodotto, essa, anche senza che confusione nè derivi, $\dot{e}$ attentato all'avviamento che è frutto dell'altrui lavoro.

L'importanza e la complessità di questo lavoro, dal punto di vista tecnico e commerciale, il valore economico rilevantissimo dei suoi resultati, è in relazione non solo alla difficoltà dei problemi tecnici risoluti, ma agli sforzi per il primo collocamento commerciale con garanzia di durevole impiego e di sempre più larga diffusione dopo superata la difficoltà dell'adozione.

Anche la unicità dell'acquirente - (quando cioè un prodotto sia realizzato per le specifiche esigenze di un sol cliente p.es. nel caso che fu più famoso in Italia quello della creazione di tipi di carri destinati ai diversi servizi militari per la motorizzazione dell'esercito) (24) se può quindi escludere la confusione, non esclude però il pericolo e il danno della imitazione servile, tanto più in quanto si tratti di un prodotto realizzato secondo le esigenze specifiche di quell'acquirente che può essere indotto ad abbandonare il suo primo fornitore e a rivolgersi al nuovo che, non avendo sostenuti le spese di preparazione potrà fare una terribile concorrenza di prezzi.

Ben più che nella semplice possibilità di confusione sull'origine del prodotto, che può determinare lo sviamento della clientela, si ha in questo caso la sottrazione precisa e voluta di un cliente determinato, che è fatto anche più diretamente contrario agli "usi onesti del commercio" e indebito sfruttamento delle spese, degli studi altrui, diretti

(24) v. le sentenze della Corte d'Appello di Genova 7 luglio 1937, in Riv. dir. priv. 1938. II. p. 1 con nota Goldschmidt, e Cáass. 17 febbraio 1938 in Riv. dir. priv. 1938. II. 89. 
ad assicurarsi un cliente avendo trovato il modo di soddisfare le specifiche esigenze.

4. Si è già rilevato come l'origine di questa teoria che vuol repressa come illecita l'imitazione servile, ed è legata ai più moderni sviluppi della tecnica, si ritrovi nella dottrina e nella giurisprudenza germanica. Ed è anche chiaro che ciò dovesse essere, in relazione al singolare rigore con cui nell'ordinamento tedesco si addiviene, previo scrupoloso esame preventivo, alla concessione dello attestato. Quanto meno riesce possibile ottenere, come in altri sistemi, facili brevetti, tanto più è sentita la necessità di assicurare una difesa a quelle attività inventive che non potessero andare coperte dal legale monopolio del brevetto. Si sono con precisati alcuni concetti perchè il principio sia contenuto nei giusti limiti. II concetto è ben nettamente precisato e chiarito dagli stessi vocaboli: non si parla dagli scrittori tedeschi di "true Aehenlichkeit", ma si dichiara illecito: "der sklavische Nachbau"!

Somiglianza anche fedele, non è imitazione, e tanto meno imitazione servile. La imitazione è lo sforzo intenzionale per conseguire somiglianza e la stessa imitazione non è la imitazione servile" ("der sklavische Nachbau"!) L'accento logico di questa locuzione, cade sul servile ("sklavische").

Fedele è una qualità, spesso una lode: servile è aggettivo di disprezzo. La fedeltà porta una divisa od una toga, il servilismo porta una livrea. Noi saremo fedeli ad un giuramento, ad una promessa ad un affetto: servili mai!

Ora il carattere tipico della imitazione servile, consiste precisamente in ciò: che la imitazione diventa riproduzione meccanica del complesso e dei dettagli, da cui esula "ogni elemento di elaborazione individuale" (come dice la Cassazione italiana nella sentenza 19 febbraio 1935 (25), - senza che l'imitatore sia neppure in grado di sceverare.

(25) v. Foro It. 1935. I. 774. 
l'essenziale dall'accessorio, quello che è così perchè deve essere così, e quello che è cosi, ma che potrebbe essere altrimenti, senza che l'imitatore si sia reso conto del perchè l'oggetto imitato od un congegno abbianno determinate caratteristiche. L'imitatore servile copia quello che è; così, come è; il congegno viene imitato per evitare lo studio, lo" sforzo, le spese, di chi quel risultato ha già conseguito.

Questo è proprio e caratteristico della imitazione servile, dove la qualifica di servile bolla appunto una imitazione: senza dignità e senza intelligenza.

In questo concetto della imitazione servile, il caratere. di illecito è indubbiamente implicito, come attentato all'avviamento della azienda del concorrente, così come è implicita la riprovazione morale come di atto "contrario agli: usi onesti del commercio" e più propriamente a quegli usi onesti dell'industria troppo dimenticati, ma richiamati espressamente dall'art. 10 bis della Convenzione internazionale.

La concorrenza sleale è diretta a reprimere ogni attentato all'avviamento del concorrente, e l'avviamento va inteso come quella possibilità di lucri futuri che deriva non solo dagli elementi singoli componenti l'azienda, ma dalla sua stessa organizzazione, la clientela è solo una delle manifestazioni esteriori dell'avviamento sia essa clientela attualle già acquisita alla azienda sia essa solo potenziale (26).

Ora la imitazione servile è attentato diretto ai risultati. di quella organizzazione industriale che costituisce parte precipua dell'avviamento di una azienda produttiva, in quanto chi servilmente imita si appropria quelle possibilità di lucri futuri che sono inerenti alla conseguita soluzione di un problema tecnico, e indirettamente si appropria quindi gli studi, gli sforzi, le spese necessarie per arrivare a quel risultato. Per tale motivo l'imitatore servile

(26) v. i miei scritti già ricordati e sopratutto: Diritto industriale, p. 415 segg. 
sompie anche un attentato alla clientela, e non solamente alla clientela possibile o potenziale, ma a quella clientela attuale della azienda concorrente che ha già conosciuto ed apprezzato un tipo ed a quel vuol restare fedele.

Se è illecita quella imitazione che può indurre in errore, che può sviare una possibile clientela, tanto più illecita è la imitazione in quanto tenta di sostituire presso il cliente o i clienti del concorrente, il prodotto da lui faticosamente realizzato, con quello che non è se non la meccanica, sfacciata copiatura del primo. Come fonte di confusione, la imitazione crea il pericolo di un danno, può distogliere, sviare per la strada il possibile cliente, ma la imitazione servile, intesa come si è detto, crea di per sè inevitabilmente il dano; ogni cliente, ogni affare che per tale via si a acquisisca, è direttamente strappato al concorrente: cosa tanto più sleale, se il prodotto sfacciatamente copiato é offerto a domicilio ed all'unico possibile cliente, ed è offerto come talora accade - da chi prestò la propria opera rinumerata alla realizzazione di quei prodotti che ora vuol soppiantare. Nel caso già ricordato di cui si è occupata la giurisprudenza italiana, era la fabbrica che aveva eseguiti sui disegni avuti da un cliente $i$ vari tipi di carri destinati ai vari usi dell'esercito che, avendoli riprodotti per proprio conto, si era offerta di eseguirli e li aveva eseguiti in grande scala quando l'amministrazione militare aveva bandito appalti per tali ingenti forniture (27).

La confusione potrà sviare il cliente: la imitazione fatta in questa forma equivale ad andare a prendere il cliente. del concorrente là dove si trova, per sottrarlo a chi già lo aveva conquistato coi suoi sforzi e con la bontà delle sue realizzazioni.

Il carattere illecito della imitazione servile tanto più si appalesa poi quando l'imitazione è fatta nei confronti non di chi genericamente ha realizzato un prodotto, ma

(27) v. App. Genova 7 luglio 1937 e Cass. 17 febbraio 1938, : sopra cit. 
*da chi si è preoccupato della soluzione di un problema specifico su espressa richiesta di un commitente, che doveva anche essere l'unico possibile acquirente.

Il carattere illecito della imitazione viene qui indubbiamente in luce sotto un altro rilfesso. La imitazione cioè, costituisce in questo caso un illecito obbiettivo, anche indipendentemente dal concorso dell'elemento subbiettivo, e dal fine doloso, e rende per ovvio riflesso, indubbiamente sleale la concorrenza attuata con questo mezzo che è di per sè obbiettivivamente illecito, come violazione deI diritto d'autore che la più parte delle legislazione positive riconosce, sui progetti e sui disegni d'ingegneria. (28).

Come sarebbe indubbiamente illecita l'opera di chi si offrisse per tradurre in esecuzione il disegno della costruzione o del piano regolatore di altro architetto, quando beninteso il committente non se ne sia espressamente assicurata la disponibilità, ma l'abbia ordinato solo facendo balenare la speranza della futura esecuzione, od abbia corrisposto solo un tenue compenso per spese materiali, tanto più è da ritenere illecita la imitazione nel caso in cui Je ideazioni tecniche e costruttive non solo avevano preso corpo in un progetto di massima od in un disegno, ma si erano concretate fin nei più minuti particolari in unità espressamente destinate a contituire dei prototipi o dei modelli! Se è illecito copiare un progetto altrui, tanto più illecito sarà copiare un modello già realizzato, quel prototipo che nella moderna produzione industriale in serie, contiene in sè potenzialmente, l'imenso valor di tutta la produzione futura.

La imitazione servile è quindi sempre e di per sè, e tanto più in questo caso, un atto di concorrenza sleale.

(28) Per la legge italiana del 22 aprile $1941 \mathrm{n} .^{\circ} 633$ si riconosce *all'autore di progetti di lavori di ingegneria che costituiscano soluzioni originali di problemi tecnici, oltre al diritto esclusivo della loro riproduzione, il diritto ad un equo compenso a carico di coloro che realizzino il progetto tecnico a scopo di lucro e senza iil suo consento (art. 99.) 
5. Nè si dica che attraverso la dottrina della repressione della imitazione servile, si estende arbitrariamente la tutela caratteristica del brevetto arrivando alla conseguenza, che si qualifica come assurda, di concedere a prodotti o meccanismi non brevettati e non brevettabili, una tutela pari per intensità, e più duratura di quella che ì concessa con conferimento del brevetto.

$\mathrm{E}$ ' evidente l'equivoco. Altra è la tutela dell'invenzione brevettata, altra è la difesa contro la concorrenza sleale.

La tutela della privativa è limitata nel tempo, ma ha come unico presupposto la esistenza di un valido brevetto, e come conseguenza la possibilità del sequestro e della distruzione dei prodotti contraffatti, oltre al risarcimento dei danni eventuali, e le possibili rigorose sanzioni penali: essa non richiede prova di colpa o di dolo (29).

L'azione per concorrenza sleale, che discende dalla imitazione servile, non è circoscritta nel tempo, ma è subordinata per il suo esercizio alla dimostrazione del dolo, anche se questo è implicito nella imitazione che pressuppone la intenzionalità (30).

Del resto, la diversità della tutela conseguibile attraverso l'azione di contraffazione e quella di concorrenza sleale si manifesta sotto un ben chiaro profilo. L'attestato

(29) Com'è noto l'azione di concorrenza sleale ̀̀ sorta nella giurisprudenza come azione di risarcimento, fondata sul principio generale della responsabilità extra contrattuale, e, come diceva iI nome, in relazione ad un comportamento doloso nell'esercizio del commercio. L'azione acquistò poi una ben più vasta funzione ed éstenzione, con uno scopo non più solo di repressione e di risarcimento danni ma di inibizione, e per tale aspetto e a tali fini i presupposti soggettivi della colpa o del dolo non apparvero più indispensabili. Ciò non toglie che certe attività di concorrenza si reprimano in quanto intenzionali e dolose come sarebbe nel caso della imitazione servile, là dove la semplice casuale somiglianza non darebbe luogo a represione o a inibizione, diversamente da quello che si verificherebbe p. es. per omonimia, anche non intenzionale, di ditta. 
di privativa garantisce il monopolio della invenzione nei confronti di chiunque arrivi anche con sforzi proprii $e$ prettamente originali allo stesso risultato, l'azione di concorrencia sleale non garantisce che altri possa arrivare indipendentemente a conseguire lo stesso risultato che non fu coperto da privativa, ma garantisce che altri non si avvalga degli studi, degli sforzi e delle spese del concorrente, realizzando atraverso una meccanica riproduzione, lo stesso prodotto. Se ci fosse concesso di ricorrere ad una metafora suggerita dalla stessa etimologia della concorrenza, e dal carattere agonistico delle competizioni commerciali, vorremmo dire che il brevetto sbarra definitivamente la strada verso il raggiungimento di quella realizzazione industriale che ne è coperta: se il brevetto non c'è la strada è libera a tutti, ma nessuno presuma di farsi rimorchiare da altri concorrenti, chi lo tentasse sarebbe nella gara squalificato o penalizzato; fuori di metafora, cadrebbe sotto le sanzioni di legge.

In queste conseguenze, checchè si dica ex adverso, non c'è niente di assurdo o di mostruoso. Sono concetti che rispondono alla più ovvia coscienza etica e giuridica, nè mancarono di avere eco in giudicati; nò si obbietti che un tal principio non sia nella coscienza degli industriali e dei commercianti: nelle coscienze degli uomini onesti e degli onesti commercianti - e sono gli uomini onesti quelli che decidono in materia di concorrenza - per fortuna nostra, sì!

Proprio un esempio suggerito dal Prola-Caselli, cade perfettamente in termini, quello dei modelli di sartoria.

Chi acquista o si fa fare da una sartina il vestito sul modello realizzato da una delle grandi case di mode francesi o italiane sa benissimo di non acquistare dall'originario creatore. Nessun pericolo di confusione vi è sull'origine del prodotto, ma non per questo si ritiene meno illecito il fatto di copiare i modelli di sartorie altrui, ed indipendentemente dallo stesso deposito nelle forme richieste dei mo- 
delli di fabbrica, la copiatura si ritenne repressa dalle leggi civili e dalla leggi penali.

Ma restando nel campo delle costruzioni automobilistiche, vorremmo sapere quale sia quel giudice o quella persona onesta che riterrebbe lecito da parte di una casa concorrente la servile imitazione di un qualsiasi tipo di vettura via via messo a punto e lanciato dalle grandi icase di produzione di automobili, che pure, di regola non sarà brevettabile ne coperto, comunque, da uno specifico diritto di privativa!

6. Per concludere, adunque l'imitazione di un prodotto non brevettato non è perciò solo sempre ed incondizionatamente lecita, essa diventa illecita anzitutto quando - qualunque sia la natura del prodotto - l'identità delle caratteristiche esteriori del prodotto sia intenzionalmente voluta ed idonea a creare confusione sull'origine del prodotto: caso questo assai frequente nella giurisprudenza.

Ma è illecita l'imitazione se altresì quando, trattandosi di una realizzazione industriale organica e complessa, essa si estenda in modo servile a tutti $i$ dettagli, in guisa che il concorrente imitatore si approprii i risultati di quel lavoro complesso e dispendioso che conduce, attraverso la combinazione degli elementi svariati - brevettati o meno alla creazione del tutto in corrispondenza alle esigenze di un particolare scopo.

Particolare carattere di illiceità riveste poi l'attività del concorrente in quei casi in cui l'imitazione si porta su prodotti che furono studiati, messi a punto e realizzati in definitivi modelli ai fini di una produzione standardizzata secondo le esigenze peculiari di un singolo cliente; su quei prodotti che non sono fine a sè stessi, ma prototipi o modelli diretti ad uno sfruttamento successivo attraverso quelle ordinazioni in serie che sono assicurate dalla buona riuscita degli esperimenti. 
Se la imitazione servile ̀̀ giustamente repressa in quanto può, determinando confusione, sviare una potenziale clientela del concorrente agendo sulla massa indistinta, del pubblico, tanto più pericolosa, dolosa, illecita e da severamente reprimersi, è quella imitazione che porta ad offrire all'unico possibile cliente quel prodotto che per lui - proprio per lui, e solo per lui - altri ha, per precedenti rapporti intercorsi, faticosamente e dispendiosamente realizzato.

In questo caso il creatore del prodotto imitato, è ben più che un commerciante un progettista. Egli ha dedicato il suo studio alla soluzione di problemi specifici a lui sottoposti ed il suo studio, le sue spese, le sue fatiche, non potranno essere ripagate che dalla successiva esecuzione, nella produzione in serie.

Il concorrente che a questo punto si insinua tra chi ha risolto il problema sottopostogli e sta per trarne $i$ frutti, e l'unico possibile acquirente, compie atto squisitamente sleale e certamente contrario a quegli "usi onesti in materia di industria o di commercio" che la Convenzione dell'Aja richiama come decisiva descriminante d'ogni attività lecita o illecita sul terreno della libera competizione commerciale. $\mathrm{E}$ ciò anche a prescindere dal diretto ed abusivo impegna di segreti o disegni del concorrente, dallo storno di collaboratori, dalla denigrazione o dalle altre circostanze che, mentre di per sè configurano nuove responsabilità a titolo di concorrenza sleale, possono particolarmente colorire l'illecito della imitazione servile. 